\title{
Optical coherence tomography angiography features of choroidal neovascularization secondary to angioid streaks
}

\author{
T. Chapron ${ }^{1}$ G. Mimoun ${ }^{1,2} \cdot$ A. Miere ${ }^{1}$ M. Srour ${ }^{1} \cdot$ A. El Ameen ${ }^{1} \cdot$ O. Semoun ${ }^{1} \cdot$ E. H. Souied ${ }^{1,3}$
}

Received: 25 January 2018 / Revised: 25 July 2018 / Accepted: 7 August 2018 / Published online: 24 September 2018

(c) The Royal College of Ophthalmologists 2018

\begin{abstract}
Purpose To characterize the features of choroidal neovascularization (CNV) secondary to angioid streaks (AS) with optical coherence tomography angiography (OCT-A) and to assess its sensitivity in CNV detection in this particular context.

Methods Consecutive patients, both with treatment-naïve and recurrent CNV associated with angioid streaks were prospectively analyzed. All patients underwent macular imaging by fluorescein angiography (FA), indocyanine green angiography (ICGA), spectral-domain (SD)-OCT, and OCT-A (AngioVue, Optovue, Optovue Inc., Freemont, CA, USA). OCT-A detection rate of $\mathrm{CNV}$ associated to AS was evaluated by two independent observers. We studied the association between OCT-A feature and either exudative or active status using Fisher exact test.

Results A total of 32 eyes of 18 consecutive patients were included in the analysis. OCT-A was able to detect CNV associated with angioid streaks in $87.5 \%$ (28/32) eyes. OCT-A phenotypes of CNV were classified into interlacing pattern in 9 eyes, pruned vascular tree pattern in 7 eyes, and combined pattern in 12 eyes (Interuser agreement: $0.871 \pm 0.071$ ). CNV were not detectable in $4 / 32$ eyes. There was a statistically significant association between the presence in OCT-A of densely ramified networks with both previous treatment status in the last 6 months $(p<0.001)$ and with exudative signs on SD-OCT $(p=0.014)$.
\end{abstract}

Conclusion OCT-A appears as a sensitive tool for detection of CNV secondary to AS. The interlacing pattern was significantly associated with active and exudative features.

\section{Introduction}

Angoid streaks (AS) are the clinical signs of extended disruptions of the Bruch membrane, which becomes calcified and fragile, allowing ingrowth of choroidal neovascularization $(\mathrm{CNV})$ into the subretinal space. AS are a major source of visual impairment, especially in middle-aged active patients, and can be associated with systemic diseases, such as pseudoxanthoma elasticum (PXE) [1]. At fundus examination, AS-which are typically bilateralharbor the aspect of linear streaks, irregularly extending

E. H. Souied

eric.souied@chicreteil.fr

1 Department of Ophthalmology, Centre Hospitalier Intercommunal de Créteil, Université Paris XII, Créteil, France

2 Centre Ophtalmologique d'Imagerie de l'Ecole Militaire, Paris, France

3 University of Paris-Est-Créteil-Val-de-Marne, Créteil, France from a peripapillary ring in a centrifugal manner [2]. In fluorescein angiography (FA), AS appear either as hyper or hypofluorescent, depending on the underlying choriocapillaris status [2]. In indocyanine green angiography (ICGA), AS appear as hypercyanescent lesions on the late phase of the examination $[1,3]$. Type $2 \mathrm{CNV}$ secondary to AS are present in $72-86 \%$ of individuals [2-5]. The type 2 $\mathrm{CNV}$ often originate in the interpapilomacular region, followed by progression towards the foveolar region [2]. Type $2 \mathrm{CNV}$ have been described by Gass as a well defined hyperfluorescent lacy pattern on FA with early staining and progressive leakage [2]. They appear on SD-OCT as a hyperreflective subretinal lesion, situated above the retinal pigment epithelium (RPE) [6-9].

With the advent of anti-VEGF therapy, a new standard of treatment appears for CNV complicating age-related macular degeneration (AMD), as well as other macular diseases such as pathological myopia or angioid streaks, consequently improving the functional outcome of these patients [10-14]. Multimodal macular imaging, including SD-OCT, FA, and ICGA is currently used to determine 
CNV morphology and activity. However, detection of CNV secondary to AS can be challenging, as the visualization of dye leakage from a neovascular lesion on FA is difficult, due to low contrast with the hyperfluorescent streaks. Moreover, both CNV and streaks are hypercyanescent in the late phase of ICGA [2, 3]. SD-OCT allows a depth resolved imaging of CNV secondary to AS, but the latter have few exudative signs and alteration could remain discrete [10].

Optical coherence tomography angiography (OCT-A) is a new imaging technique allowing visualization of both retinal and choroidal microvascularization, as well as abnormal vessels, in a noninvasive manner. It is an en-face derived technique, based on the split-spectrum amplitudedecorrelation angiography algorithm revealing dynamic motion of erythrocytes [15]. This new technique may be used in daily clinical ophthalmology practice and may in the future replace invasive techniques.

This study was designed to report the morphological features and the sensitivity of OCT-A in the detection of CNV secondary to AS.

\section{Methods}

We included in this prospective study all patients diagnosed with AS complicated by $\mathrm{CNV}$ at the Eye University of Creteil (University Paris-Est) and at the Ecole Militaire Retinal Center between November 2014 and November 2015. This study was performed according to the French bioethical legislation and the Declaration of Helsinki for research involving human subjects. A written informed consent was obtained for all patients.

Inclusion criteria were patients diagnosed with angioid streaks associated to $\mathrm{CNV}$, as demonstrated on the basis of FA + ICGA and SD-OCT examinations. On FA, the diagnosis of CNV secondary to AS was made on a lacy pattern with early staining and progressive leakage in the late frames. On ICGA, CNV secondary to AS harbored the aspect of a hypercyanescent lesion in the late frames of the examination. On SD-OCT, type $2 \mathrm{CNV}$ lesions were confirmed as a hyperreflective lesion situated above the RPE, corresponding to the neovascular membrane, accompanied or not by subretinal and intraretinal fluid [16]. CNVs activity was defined by either recent macular hemorrhages neighboring a known CNV or a late leakage on FA or a hyperfluorescent late lesion on ICGA or intraretinal or subretinal fluid in SD-OCT or more than $10 \%$ variation of retina thickness in SD-OCT compared to last examination [10]. If none of these signs were found, CNV was considered as nonexudative. All images were reviewed for final inclusion by a senior physician (G.M.).

Exclusion criteria consisted of patients with prior vitroretinal surgery or other confounding ocular disease such as neovascular and atrophic AMD, adult-onset foveomacular vitelliform dystrophy, diabetic retinopathy, chronic central serous chorioretinipathy, high myopia or posterior uveitis.

All patients underwent a complete ocular clinical exam including best corrected visual acuity, using early treatment diabetic retinopathy study charts, fundus biomicroscopy as well as multimodal imaging including infrared imaging, autofluorescence photographs, SD-OCT, FA, ICGA (Spectralis, Heidelberg Retinal Analyzer, Heidelberg Engineering, Inc., Heidelberg, Germany) and OCT-A (RTVue XR Avanti, Optovue Inc., Fremont California, USA). Data about age, gender, medical history, including PXE status confirmed by skin biopsy, duration of the disease (AS and $\mathrm{CNV}$ ), and previous treatment status were recorded.

The OCT-A instrument used in our study has an A-scan rate of 70,000 scans per second, using a light source centered on $840 \mathrm{~nm}$ and a bandwidth of $50 \mathrm{~nm}$. Each OCT-A volume contains $304 \times 304$ A-scans with two consecutive B-scans captured at each fixed position before proceeding to the next sampling location. Split-spectrum amplitude-decorrelation angiography was used to extract the OCT-A information. Each OCT-A volume was acquired in $3 \mathrm{~s}$, and two orthogonal OCT-A volumes were acquired to perform motion correction to minimize motion artifacts arising from microsaccades and fixation changes. Angiography information displayed is the average of the decorrelation values when viewed perpendicularly through the thickness being evaluated. Automatic retinal segmentation was performed by embedded software in the machine, sometimes necessitating a slight adjustment from the clinician for two eyes. A $3 \times 3 \mathrm{~mm}$ and $6 \times 6 \mathrm{~mm}$ scanning area centered by the CNV or the fovea were performed. The CNV area was manually measured in the choriocapillaris segmentation $(30-60 \mu \mathrm{m}$ underneath the RPE), using two features embedded in the instrument: "select area" and "vessel area". Two masked observers assessed the presence and activity of CNV on FA, ICG, and SD-OCT imaging (GM and MS) and two other masked observers (TC and AM) independently analyzed OCT-A images alone, in order to identify the presence of $\mathrm{CNV}$ and to describe the features of the neovascular lesion. In case of disagreement, open adjustments were realized.

We assessed the presence of several criteria in order to characterize the neovascular membranes on OCT-A images, based on current literature [17-21], such as the high flow, densely ramified, convoluted vascular network with thin capillaries for which we coined the term "interlacing" vascular network; "pruned vascular tree" vascular network was defined by a filamentous flow with persistence of main vascular trunks, but no thin ramifications. The "mixed" vascular network consisted of combined characteristics of the above-described patterns. The presence/absence of a dark halo was also assessed. 


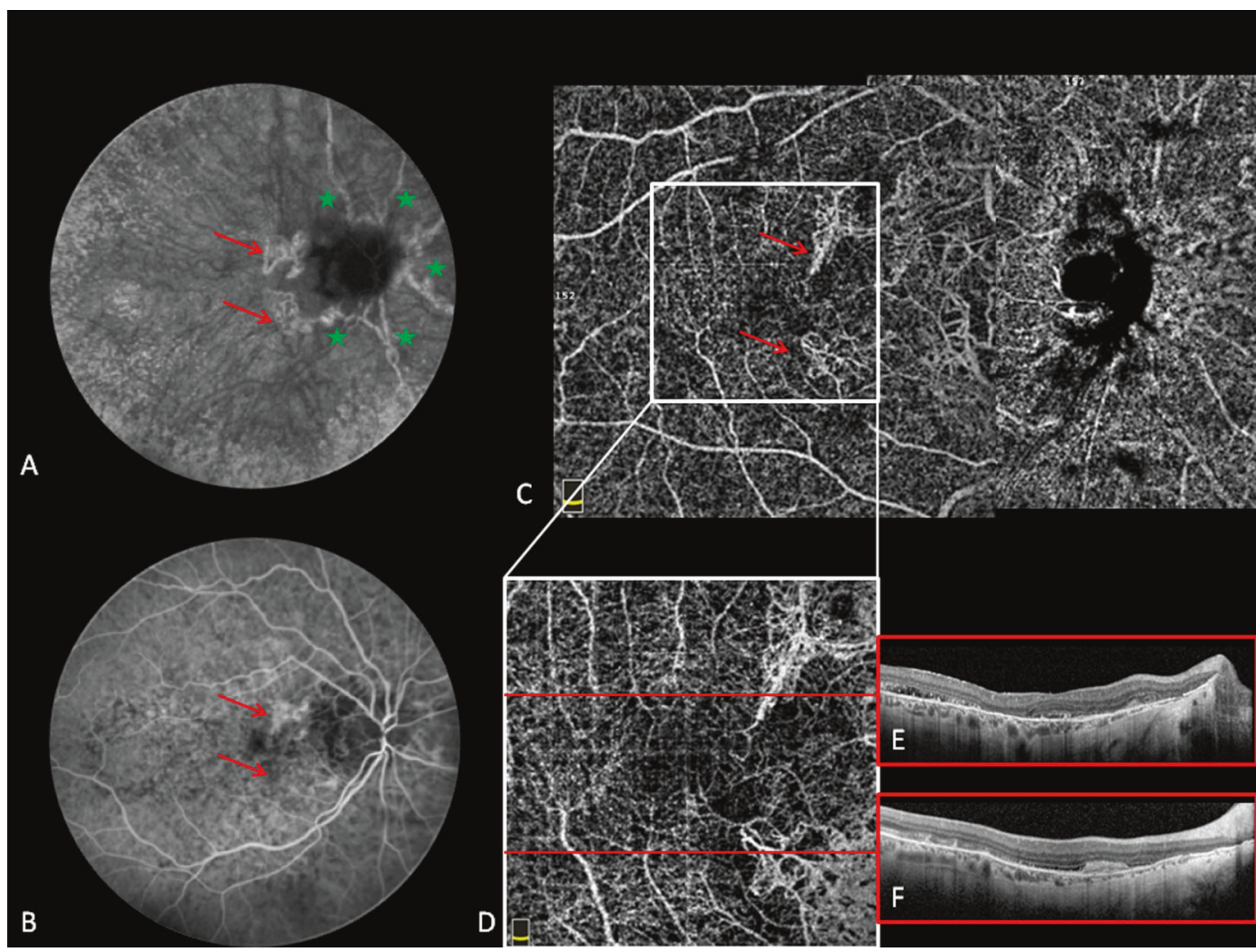

Fig. 1 Multimodal imaging of the right eye of a 58-years-old patient with Angioid Streaks and secondary choroidal neovascularization. Upper left panel: late frame indocyanine green angiography (ICGA) reveals two hyperfluorescent late interpapillomaculary lesions (red arrow) and hyperfluorescent streaks (green stars). Lower left panel: Late frame fluorescein angiography (FA) reveals a hyperfluorescent lesion in the superior interpapilomaculary area (red arrow), while the corresponding inferior interpapillomaculary area is not visible. Upper right panel: $6 \times 6 \mathrm{~mm}$ optical coherence tomography angiography

Statistical analysis was performed using the Statistical Package for Social Sciences (version 22.0; SPSS, Inc., Chicago, IL, USA). The analysis included descriptive statistics for demographics and main clinical data and qualitative descriptions of the imaging characteristics. Categorical variables were compared using the Fischer exact and quantitative data were compared using the ANOVA test. Coefficient of correlation of Spearman were use to study the association between OCT-A feature and both exudation feature on multimodal imaging and previous treatment received. $P=0.05$ was considered to be the threshold for significance.

\section{Results}

We included 32 eyes of 18 consecutive patients ( 11 men and 7 women, mean age $59.7 \pm 8.7$ years, range from 40 to 71 years) with CNV secondary to AS. 14 patients presented a PXE disease confirmed by skin biopsy, no patient
(OCT-A) composite image showing, on the manual $30 \mu \mathrm{m}$ segmentation around Bruch's membrane, two high flow, interlacing neovascular lesions (red arrow). Lower middle panel: $3 \times 3 \mathrm{~mm}$ OCTAngiography reveals, on the manual $30 \mu \mathrm{m}$ segmentation around Bruch's membrane, two highly structured, interlacing neovascular lesions (red arrow). Lower right panel: spectral-domain optical coherence tomography (SD-OCT) passing through the superior (top) and inferior (bottom) intermaculopapillary lesion

presented haemoglobinopathy or Paget disease. An example of the FA, ICG, and OCT-A imaging performed on these patients is presented in Fig. 1.

Demographical data of patients is presented in Table 1. Concerning the $\mathrm{CNV}$ localization in the macula, in our cohort $62.5 \%$ (20/32) eyes were situated subfoveally, $15.6 \%$ (5/32) juxtafoveally, $18.8 \%$ (6/32) interpapilomaculary, and $3.1 \%(1 / 32)$ juxtapapilary. Out of 32, 28 eyes in our series had previously received antivascular endothelial growth factor (VEGF) therapy, with mean intravitreal injections of $10.97 \pm 9.4$. Moreover $1 / 32$ eye had previously received macular laser and photodynamic therapy. Finally, 3/32 eyes were treatment-naïve.

On fundus biomicroscopy, macular hemorrhage was observed in only $1 / 32(3.1 \%)$ eyes. On FA, 9/32 eyes (28.1\%) presented a late leakage. ICGA delineated a hypercyanescent plaque in 22/32 eyes (68.75\%). On SDOCT, a hyperreflective lesion corresponding to type $2 \mathrm{CNV}$ scarring tissue was present in 32/32 eyes (100\%), accompanied by the presence of subretinal fluid in $4 / 32$ eyes 
(12.5\%), and/or intraretinal fluid in 8/32 eyes (25\%) [20]. Based on multimodal imaging, $\mathrm{CNV}$ were classified as exudative lesions in 12/32 (37.5\%) eyes at inclusion. A total of $14 / 32$ eyes $(43.7 \%)$ did not received anti-VEGF treatment within the last 6 months.

OCT-A was able to detect CNV in 28/32 (87.5\%) eyes in the choriocapillaris segmentation. The CNV network complicating AS, as confirmed by multimodal imaging, could not be detected in $4 / 32$ eyes. The two OCT-A readers agreed on the presence or absence of $\mathrm{CNV}$ in all eyes.

Table 1 Demographical data of included eyes with choroidal neovascularization secondary to angioid streaks

\begin{tabular}{ll}
\hline Age $($ mean \pm SD) & $59.7 \pm 8.7$ \\
Sex, $n(\%)$ & \\
Men & $22(68.6)$ \\
Women & $10(45.4)$ \\
Mean duration of follow-up (years \pm SD) & $9.97 \pm 17.2$ \\
Previous treatment, $n(\%)$ & \\
IVI & $28(87.5)$ \\
PDT and/or laser & $9(28.1)$ \\
Naive & $3(9.4)$ \\
Localization, $n(\%)$ & \\
Foveolar & $20(62.5)$ \\
Juxta-foveolar & $5(15.6)$ \\
Interpapillo-macular & $6(18.8)$ \\
Juxta-papillar & $1(3.1)$ \\
PXE status, $n(\%)$ & $25(78.1)$ \\
No injection in the past 6 months, $n(\%)$ & $14(43.75)$ \\
\hline
\end{tabular}

$S D$ standard deviation; IVI intravitreal injection; $P D T$ photodynamic therapy;

$P X E$ pseudoxanthoma elasticum
Quantitative analysis of the 28 eyes in which OCT-A detected a high flow neovascular network, showed that the lesion area ("select area") ranged from 0.22 to $9.67 \mathrm{~mm}^{2}$ (mean $2.24 \pm 2.59$ ). "Vessel area", accounting only for the vasculature automatically detected within the lesion area, ranged from 0.13 to $6.77 \mathrm{~mm}^{2}$ (mean $1.53 \pm 1.79$ ).

Qualitative evaluation of CNV complicating AS revealed distinct phenotypes of neovascular membranes. Finally, we identified 9 eyes as "interlacing" vascular network (Fig. 2, $28.1 \%, 9 / 32$ eyes) and 7 eyes as "pruned vascular tree" (Fig. 3, 21.9\%, 7/32 eyes). In 12/32 eyes (37.5\%, Fig. 3, Right panel), we found combined features of the abovedescribed phenotypes. Moreover, 6/32 eyes (18.75\%) presented a dark area surrounding the neovascular complex, for which we coined the term "dark halo". All eyes that presented a dark halo received an anti-VEGF treatment in the past 6 months $(p=0.024)$ and $4 / 6$ eyes $(66.7 \%)$ had exudative signs $(p=0.165)$. Statistical correlations between the activity status (as assessed by the presence of exudative signs on conventional multimodal imaging) and the OCT-A phenotype of the CNV were performed. Using the Fisher exact test, we have found a statistically significant association between antiangiogenic treatment and pattern type, as follows: recent anti-VEGF treatment $(<6$ months) was associated with both the interlacing and combined patterns, while in the eyes without treatment in the last 6 months the pruned vascular tree pattern was prevalent $(p<0.001)$. Statistical analysis (Fisher exact test) revealed an association between exudative signs on multimodal imaging and pattern type $(p=0.014)$. Statistical results are shown in Table 2. Moreover, lesion area, as measured with the "select area" and "vessel area" features of the software, were not associated with pattern type $(F=0.734, p=0.54$ and $F=$
Fig. $23 \times 3$ Optical coherence tomography angiography (OCTA) and corresponding B-scan of two eyes presenting a interlacing pattern of choroidal neovascularization secondary of AS. Right and left panels reveal high flow, densely ramified, convoluted vascular networks. Note the presence of a dark halo (red arrow)
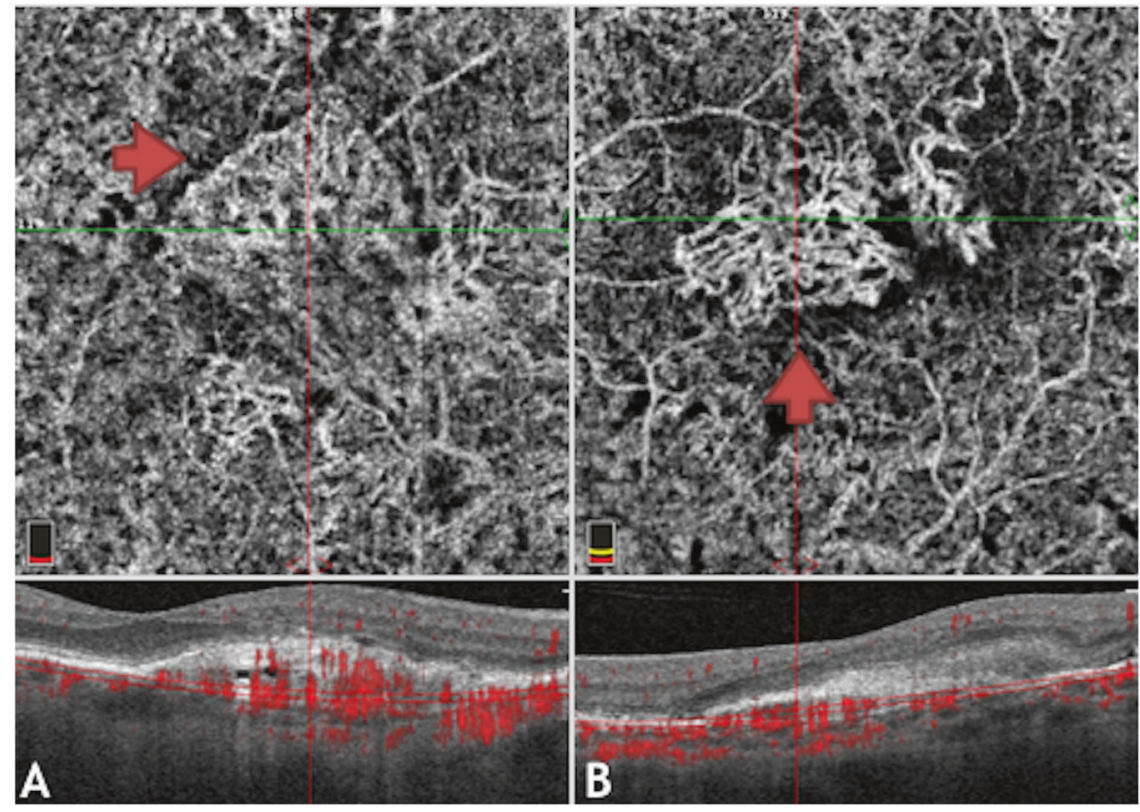
Fig. $33 \times 3$ Optical coherence tomography angiography (OCTA) and corresponding B-scan of two eyes presenting a pruned vascular tree and a mixed pattern of choroidal neovascularization secondary of AS imaged by OCT-A. OCT-A revealed in both the left and right panels a high flow network with filamentous flow and no thin capillaries. Note that on the right panel there is a combined pattern, showing both filamentous flow vessels (red star) and a densely packed, interlacing pattern (red arrowhead)

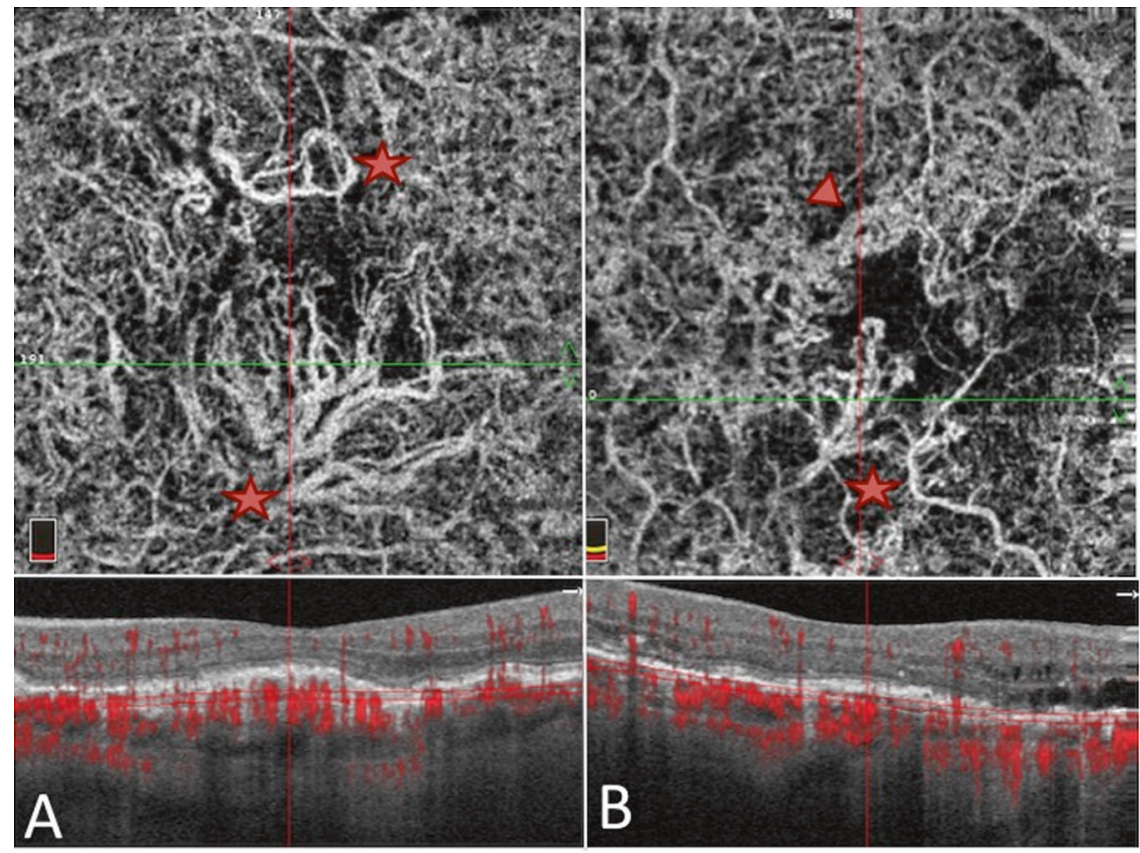

\begin{tabular}{lll}
\hline & $\begin{array}{l}\text { Presence of anti-VEGF treatment in } \\
\text { the past } 6 \text { months }(N=32)\end{array}$ & $\begin{array}{l}\text { Presence of exudative signs in } \\
\text { multimodal imaging }(N=32)\end{array}$ \\
\hline $\begin{array}{l}\text { "Interlacing" }(n=9) \\
\text { "Combined" }(n=12)\end{array}$ & 8 & 5 \\
"Pruned vascular tree" $(n$ & 0 & 7 \\
$=7)$ & 0 \\
$\begin{array}{l}\text { Not detected in OCT-A }(n \\
=4)\end{array}$ & 0 & 0 \\
$p$ & $<0.001$ & 0.014 \\
\hline
\end{tabular}

The obtained $p$ shows the statistical association of pattern with an anti-VEGF treatment in the past 6 months and with the presence of exudative signs in multimodal imaging
Table 2 Optical coherence tomography - angiography (OCT-A) morphological features of choroidal neovascularization (CNV) secondary to angioid streaks (AS) in eyes having received intravitreal treatment in the past 6 months and with exudative signs in multimodal imaging respectively, using Fisher exact test current literature [20, 22]. However, for 4/32 eyes, OCT-A was unable to detect the neovascular membrane, related to low-image quality, due to eye movement secondary to the lack of fixation (update software with eyetracking not available at the time of the study). In our series, we distinguished two patterns of CNVs on OCT-A: interlacing pattern and pruned vascular tree pattern. While the interlacing, densely packed CNV were correlated in our analysis with the presence of exudative signs on SD-OCT, FA, and ICGA; the pruned vascular tree pattern consisted of large dilated vascular trunks were correlated with an absence of exudative signs $(p=0.014)$ [18]. However, in $12 / 32$ eyes the CNV morphology presented combined features. These findings suggest that CNVs pattern could provide useful insights on their activity. Moreover, a dark halo surrounding the CNV was detected in the choriocapillaris segmentation. This dark halo was correlated in a statistically significant manner with eyes having received anti-VEGF therapy in the last 6 months $(p=0.024)$. Corbeli et al. recently described CNV complicating AS and distinguishing between two Related Macular Degeneration and $90 \%$ in myopic CNV in 
patterns called "tangled" and "interlacing" [23]. Our results are consistent with their findings. Nonetheless, they did not describe the mixed pattern, consisting of features of both tangled and interlacing, which accounts for the majority of our cohort. Interestingly, we also found an association between "interlacing" pattern and exudative status.

Indeed, detection of CNVs secondary to AS in FA or in ICGA can be challenging.

OCT-A is able to detect flow movement by detecting erythrocyte motion thus overcoming the constraints of dyebased angiography. Given its high sensitivity, OCT-A may detect newly formed CNV that are not yet symptomatic and/or not well visualized in standard imaging [19, 20, 22, 24-29].

In our cohort we found a high number of combined forms, helping us to better understand the CNV lifecycle. We could hypothesize that newly formed CNVs usually appear aside a streak in the interpapilary region, with an interlacing pattern. When they secondary grow and reach the macula, they become symptomatic. The delay between the actual presence of $\mathrm{CNV}$ and visual loss explains why CNVs secondary to AS have a larger area than type $2 \mathrm{CNV}$ associated with AMD, given that the latter begins more often in the retro or juxta-foveolar region (mean $2.24 \mathrm{~mm}^{2}$ in AS versus $0.25 \mathrm{~mm}^{2}$ in AMD) [30, 31]. Concerning Type $2 \mathrm{CNV}$ complicating pathologic myopia, although CVN size is much smaller $\left(0.34 \mathrm{~mm}^{2}\right)$, the delay between $\mathrm{CNV}$ formation and vision loss is shorter, as these new vessels tend to appear in the (peri)foveolar region, generating symptoms quite quickly [32].

In time, and after repeated antiangiogenic treatment, the interlacing pattern progresses to a mature and less active neovascularization, corresponding to the pruned vascular tree. Recurrences could appear on the edge of or within the pruned vascular tree, generating a combined (interlacing + pruned) pattern [33]. In our series, this was observed in all eyes with the combined form; however, we did not observe any new CNV sprouting outside the region of a pruned $\mathrm{CNV}$. This hypothesis is comforted by the fact that there is a large variation in CNVs' size, ranking from 0.22 to 9.67 $\mathrm{mm}^{2}$ with a standard deviation of 2.59 .

Our study has multiple limitations. Type $2 \mathrm{CNV}$ has been evaluated in the choriocapillaris segmentation, where they appear due to the projection artifact. The reason for this was the fact that the "Remove artifact" option on outer retinal segmentation partially blocks signal from areas with slower flow and that, without this option, projected retinal vasculature can make image analysis confusing. Artifacts are an important limitation on OCT-A images, and they have been ruled out on both lectures by minor manual adjustments of the segmentation, whenever there were errors due to eye movement, poor fixation or changes in the retinal architecture. Furthermore, the limited sample size and heterogeneity, with a small number of treatment-naïve
$\mathrm{CNV}$, is a serious limitation, as we included both treated and treatment-naive eyes of patients with AS. As we do not have longitudinal follow-up, this study did not allow us to analyze OCT-A pattern changes and there was no control group for AS not complicated by CNV, so we cannot assess the negative predicted value. Moreover, OCT-A pattern classification is widely discussed and no consensus on the aspect (and value) of these patterns has been reached. Thus, a convergence and harmonization of the terminology itself and defining criteria for used patterns should be implemented through literature.

Indeed, larger, more homogenous cohorts need to be evaluated, in order to confirm the place of OCT-A in a clinical setting.

$\mathrm{CNV}$ activity based on morphology and lesion area, as assessed by OCT-A, is still a new field. In our study we suggested a relationship between OCT-A neovascular patterns of CNVs secondary to AS and activity status, as documented on conventional imaging. Given its high detection rate $(87.5 \%)$ and its potential help in determining prognosis, OCT-A may be thus integrated in the multimodal imaging of a patient with CNV secondary to AS.

\section{Summary}

\section{What was known before}

- OCT-A is a new non invasive technique to detect CNV type 2 for example in AMD or high myopia.

- Few articles studied the OCT-A accuracy in detecting CNV secondary to AS.

\section{What this study adds}

- In our study including consecutive patients with CNV secondary to AS, we found a detection rate of $87.5 \%$.

- The presence of densely ramified networks was associated with exudative signs in multimodal imaging and with an anti-VEGF treatment received in the past 6 months

\section{Compliance with ethical standards}

Conflict of interest The authors declare that they have no conflict of interest.

\section{References}

1. Connor PJ, Juergens JL, Perry HO, Hollenhorst RW, Edwards JE. Pseudoxanthoma elasticum and angioid streaks. A review of 106 cases. Am J Med. 1961;30:537-43. 
2. Clarkson JG, Altman RD. Angioid streaks. Surv Ophthalmol. 1982;26:235-46.

3. Mansour AM, Shields JA, Annesley WH, el-Baba F, Tasman W, Tomer TL. Macular degeneration in angioid streaks. Ophthalmol J. 1988;197:36-41.

4. Lim JI, Bressler NM, Marsh MJ, Bressler SB. Laser treatment of choroidal neovascularization in patients with angioid streaks. Am J Ophthalmol. 1993;116:414-23.

5. Singerman LJ, Hatem G. Laser treatment of choroidal neovascular membranes in angioid streaks. Retina. 1981;1:75-83.

6. Gass JD. Pathogenesis of disciform detachment of the neuroepithelium. Am J Ophthalmol. 1967;63:Suppl: 1-139.

7. Gelisken F, Inhoffen W, Schneider U, Stroman G, Kreissig I. Indocyanine green angiography in classic choroidal neovascularization. Jpn J Ophthalmol. 1998;42:300-3.

8. Hee MR, Baumal CR, Puliafito CA, Duker JS, Reichel E, Wilkins JR, et al. Optical coherence tomography of age-related macular degeneration and choroidal neovascularization. Ophthalmology. 1996;103:1260-70.

9. Semoun O, Guigui B, Tick S, Coscas G, Soubrane G, Souied EH. Infrared features of classic choroidal neovascularisation in exudative age-related macular degeneration. $\mathrm{Br} \mathrm{J}$ Ophthalmol. 2009;93:182-85.

10. Mimoun G, Tilleul J, Leys A, Coscas G, Soubrane G, Souied EH. Intravitreal ranibizumab for choroidal neovascularization in angioid streaks. Am J Ophthalmol. 2010;150:692-700.

11. Tilleul J, Mimoun G, Querques G, Puche N, Zerbib J, Lalloum F, et al. Intravitreal ranibizumab for choroidal neovascularization in angioid streaks: four-year follow-up. Retina. 2015;36:483-91.

12. Neri P, Salvolini S, Mariotti C, Mercanti L, Celani S, Giovannini A. Long-term control of choroidal neovascularisation secondary to angioid streaks treated with intravitreal bevacizumab (Avastin). $\mathrm{Br}$ J Ophthalmol. 2009;93:155-58.

13. Schiano Lomoriello D, Parravano MC, Chiaravalloti A, Varano M. Choroidal neovascularization in angioid streaks and pseudoxanthoma elasticum: 1 year follow-up. Eur J Ophthalmol. 2009;19:151-53.

14. Sawa M, Gomi F, Tsujikawa M, Sakaguchi H, Tano Y. Long-term results of intravitreal bevacizumab injection for choroidal neovascularization secondary to angioid streaks. Am J Ophthalmol. 2009;148:584-90.

15. Jia Y, Tan O, Tokayer J, Potsaid B, Wang Y, Krauss MF, et al. Split-spectrum amplitude-decorrelation angiography with optical coherence tomography. Opt Express. 2012;20:4710-25.

16. Bird AC, Bressler NM, Bressler SB, Chisholm IH, Coscas G, Davis $\mathrm{MD}$, et al. An international classification and grading system for age-related maculopathy and age-related macular degeneration. The International ARM Epidemiological Study Group. Surv Ophthalmol. 1995;39:367-74.

17. Dolz-Marco R, Phasukkijwatana N, Sarraf D, Freund KB. Regression of type 2 neovascularization into a type 1 pattern after intravitreal antivascular endothelial growth factor therapy for neovascular age-related macular degeneration. Retina. 2017;37:222-33

18. Miere A, Semoun O, Cohen SY, El Ameen A, Srour M, Jung C, et al. Optical coherence tomography angiography features of subretinal fibrosis in age-related macular degeneration. Retina. 2015;35:2275-84.
19. Miere A, Querques G, Semoun O, El Ameen A, Capuano V, Souied EH. Optical coherence tomography angiography in early type 3 neovascularization. RetinPa. 2015;35:2236-41.

20. Bis. Miere A, Butori P, Salomon YCohen, Semoun O, Capuano $\mathrm{V}$, et al. Vascular remodeling of choroidal neovascularization after antivascular endothelial growth factor therapy visualized on optical coherence tomography angiography. Retina. 2017;0:1-10.

21. Querques L, Giuffrè C, Corvi F, Zucchiatti I, Carnevali A, De vitis LA, et al. Optical coherence tomography angiography of myopic choroidal neovascularisation. Br J Ophthalmol. 2016;101:609-15.

22. El Ameen A, Cohen SY, Semoun O, Miere A, Srour M, Quaranta El Maftouhi M, et al. Type 2 neovascularization secondary to agerelated macular degeneration imaged by optical coherence tomography angiography. Retina. 2015;35:2212-18.

23. Corbelli E, Carnaveli A, Marchese A, Cicinelli MV, Querques L, Sacconi R, et al. Optical coherence tomography angiography features of angioid streaks. Retina. 2017;0:1-9.

24. Kuehlewein L, Bansal M, Lenis TL, Lafe NA, Sadda SR, Bonini Filho MA, et al. Optical coherence tomography angiography of type 1 neovascularization in age-related macular degeneration. Am J Ophthalmol. 2015;160:739-48.

25. Chan SY, Wang Q, Wei WB, Jonas JB. Optical coherencetomographic angiography in central serous chorioretinopathy. Retina. 2016;36:2051-58.

26. De Carlo TE, Rosenblatt A, Goldstein M, Baumal CR, Loewenstein A, Duker JS. Vascularization of irregular retinal pigment epithelial detachments in chronic central serous chorioretinopathy evaluated with OCT-angiography. Ophthalmic Surg Lasers Imaging Retina. 2016;47:128-33.

27. Costanzo E, Cohen SY, Miere A, Querques G, Capuano V, Semoun $\mathrm{O}$, et al. Optical coherence tomography angiography in central serous chorioretinopathy. J Ophthalmol. 2015;2015:134783.

28. Querques G, Zambrowski O, Corvi F, Miere A, Semoun O, Srour $\mathrm{M}$, et al. Optical coherence tomography angiography in adultonset foveomacular vitelliform dystrophy. $\mathrm{Br} \mathrm{J}$ Ophthalmol. 2016;100:1724-30.

29. Lupidi M, Coscas G, Cagini C, Coscas F. Optical coherence tomography angiography of a choroidal neovascularization in adult-onset foveomacular vitelliform dystrophy: pearls and pitfalls. Invest Ophthalmol Vis Sci. 2015;56:7638-45.

30. Coscas G, Lupidi M, Coscas F, Français C, Cagini C, Souied EH. Optical coherence tomography angiography during follow-up: qualitative and quantitative analysis of mixed type I and II choroidal neovascularization after vascular endothelial growth factor trap therapy. Ophthalmic Res. 2015;54:57-63.

31. Spaide RF. Optical coherence tomography angiography signs of vascular abnormalization with antiangiogenic therapy for choroidal neovascularization. Am J Ophthalmol. 2015;160:6-16.

32. Bruyère $E$, Miere A, Cohen SY, Martiano D, Sikorav A, Popeanga A, et al. Neovascularization secondary to high myopia imaged by optical coherence tomography angiography. Retina. 2017;37:2095-101.

33. Ebran J-M, Mimoun G, Cohen S-Y, Grenet T, Donati A, JeanPastor MJ, et al. Treatment with ranibizumab for choroidal neovascularization secondary to a pseudoxanthoma elasticum: results of the French observational study PiXEL. J Fr Ophtalmol. 2016;39:370-75. 